7th International Workshop on Astronomy and

Relativistic Astrophysics (IWARA 2016)

International Journal of Modern Physics: Conference Series

Vol. 45 (2017) 1760024 (9 pages)

(C) The Author(s)

DOI: $10.1142 / \mathrm{S} 2010194517600242$

\title{
White Dwarf Pulsars and Very Massive Compact Ultra Magnetized White Dwarfs
}

\author{
Edson Otoniel, R. V. Lobato, M. Malheiro \\ Departamento de Física \\ Instituto Tecnológico de Aeronáutica (ITA) \\ 12228-900 São José dos Campos, SP, Brazil \\ otoniel@ita.br,malheiro@ita.br \\ Bruno Franzon, Stefan Schramm \\ Frankfurt Institute for Advanced Studies (FIAS) \\ Ruth-Moufang-Str. 1, D-60438 Frankfurt am Main, Germany \\ franzon@fias.uni.frankfurt.de,schramm@fias.uni-frankfurt.de \\ Fridolin Weber \\ Department of Physics \\ San Diego State University (SDSU) \\ 5500 Campanile Drive, 92182 San Diego, California, USA \\ Center for Astrophysics and Space Sciences \\ University of California at San Diego, 92093 La Jolla, California, USA \\ fweber@mail.sdsu.edu
}

Published 15 August 2017

\begin{abstract}
In this work, we discuss white dwarf pulsars found recently making also reference of the possibility of some SGRs/AXPs being part of this class of pulsars. We also study the properties of very massive compact ultra magnetized white dwarfs that could be the progenitors candidates of super luminous type Ia supernovae, and also a previous stage of these white dwarf pulsars before the magnetic field decay. The structure of this ultra magnetized white dwarfs is obtained by solving the Einstein-Maxwell equations with a poloidal magnetic field in a fully general relativistic approach. The stellar interior is composed of a regular crystal lattice made of carbon ions immersed in a degenerate relativistic electron gas. We find that magnetized white dwarfs violate the standard Chandrasekhar mass limit significantly. We obtain a maximum white dwarf mass of around $2.12 M_{\odot}$ with an equatorial radius $R \sim 1596 \mathrm{Km}$, a central magnetic field of $B_{c}=1.74 \times 10^{14} \mathrm{G}$ and $B_{s}=3.6 \times 10^{13} \mathrm{G}$ at the stellar surface.
\end{abstract}

Keywords: Magnetized White Dwarfs, Poloidal Magnetic Field, Pulsars, Magnetars.

PACS numbers: 97.20.Rp, 97.60.Gb, 04.40.Dg, 98.70.Qy, 04.25.D-, 97.10.Nf

This is an Open Access article published by World Scientific Publishing Company. It is distributed under the terms of the Creative Commons Attribution 4.0 (CC-BY) License. Further distribution of this work is permitted, provided the original work is properly cited. 


\section{E. Otoniel et al.}

\section{Introduction}

Recently some massive white dwarfs with high magnetic field $\left(10^{6}-10^{9} \mathrm{G}\right)$ on their surfaces have been observed, ${ }^{1}$ and these magnetic fields are higher than normal values for white dwarfs $10^{4} \mathrm{G}$ (for a recent review see Ref. ${ }^{2}$ ). Few years ago this number has grown significantly with new observations. More recently, a white dwarf pulsar known as AR Scorpii has been discovered with few minutes of period, showing radiation in a broad range of frequencies. ${ }^{3,4}$ Another specific example is the AE Aquarii binary system formed by a very fast intermediate polar white dwarf with a short period of $P=33 \mathrm{~s}$.

It is generally accepted that stars born with masses below 10 solar masses end up their evolutions as white dwarfs (WDs). ${ }^{5}$ These stars with a typical composition mostly made of carbon, oxygen, or helium, white dwarfs possess central densities up to $\sim 10^{11} \mathrm{~g} / \mathrm{cm}^{3}$. They can be very hot, fast rotating and strongly magnetized. The internal magnetic fields of white dwarfs are not known, but they are expected to be larger than their surface magnetic fields. This is due to the fact that in ideal magneto hydrodynamics (MHD), the magnetic field, $B$, is 'frozen' with the fluid and $B \propto \rho$, with $\rho$ being the local mass density. A simple estimate follows from the virial theory by equating the magnetic field energy with the gravitational binding energy, which leads to an upper limit for the magnetic fields in the WDs surface of $\sim 10^{13}$ G. On the other hand, analytic and numeric calculations, both in Newtonian theory as well in General Relativity theory, show that WDs may have internal magnetic fields as large as $10^{12-15} \mathrm{G}$.

\section{SGRs/AXPs as Fast Rotating, Massive and Highly Magnetized White Dwarfs}

It has been proposed by Malheiro, Rueda and Ruffini an alternative model considering Soft Gamma Repeaters (SGRs) and Anomalous X-Ray Pulsars (AXPs) as white dwarf pulsars. ${ }^{6}$ SGRs/AXPs are very slow rotating stars $P \sim(2-12)$ s, with intense magnetic field of $\sim 10^{15} \mathrm{G}$ (see Ref. ${ }^{7}$ ) and with spin-down rates $\dot{P} \sim\left(10^{-13}-10^{-10}\right)$ s/s, larger than the ones of normal pulsars $\dot{P} \sim\left(10^{-15}-10^{-14}\right) \mathrm{s} / \mathrm{s}$ (see Refs. $\left.{ }^{8,9}\right)$. Their X-ray luminosities $L_{X}$, are explained by the decay of their huge magnetic fields. Currently there are $23 \mathrm{SGRs} / \mathrm{AXPs}$ that are classified as magnetars and 6 until now only candidates. ${ }^{10}$ This class of stars can be understood in terms of a canonical spin-powered pulsars model, because in several aspects they are similar. ${ }^{11-13}$ Considering a star with $M=1.4 M_{\odot}$ and $R=10^{6} \mathrm{~cm}$ (a neutron star NS), the magnetic field at poles is:

$$
B_{\mathrm{p}}^{\mathrm{NS}}=3.2 \times 10^{19}(P \dot{P})^{1 / 2} \mathrm{G} .
$$

In the case of a white dwarf with $M=1.4 M_{\odot}$ and $R=3 \times 10^{8} \mathrm{~cm}$, there is a new scale for the magnetic field at poles:

$$
B_{\mathrm{p}}^{\mathrm{WD}}=4.21 \times 10^{14}(P \dot{P})^{1 / 2} \mathrm{G},
$$


and to the dipole moment $\mu$, responsible by the dipole radiation emitted, which is a factor $10^{3}$ times larger than for neutron stars. There are also new values for the mass density, moment of inertia, and rotation energy. ${ }^{14,15}$

\section{Magnetic White Dwarf}

In the center of a white dwarf the pressure is given by the sum of the degenerate electron pressure $P_{e}$ and the lattice pressure $P_{L}$, and the energy density by the sum of the ions energy density, degenerate electron energy $\mathcal{E}_{e}$, and energy density of the ionic lattice $\mathcal{E}_{L}$

$$
\begin{aligned}
P & =P_{e}+P_{L}(Z), \\
\mathcal{E} & =n_{x} M(Z, A) c^{2}+\mathcal{E}_{e}+\mathcal{E}_{L}-n_{e} m_{e} c^{2},
\end{aligned}
$$

where $Z$ is the average proton numbers, $n_{x}$ the number densities of nuclei, and $M(Z, A)$ the experimental nuclear masses, ${ }^{16} n_{e}$ the electron number density, $m_{e}$ the electron mass, and $c$ the speed of light. The pressure of the ionic lattice is given by

$$
P_{L}=\frac{\mathcal{E}_{L}}{3},
$$

where the ionic energy density is

$$
\mathcal{E}_{L}=C e^{2} n_{e}^{4 / 3} Z^{2 / 3}
$$

with the lattice constant $C$ of a regular crystal structure of the type body-centeredcubic (bcc) is $C=-1.444231$.

\subsection{White Dwarf Pulsars}

To explain a peculiar rapid pulsating radio signal observed by the Cambridge radio astronomy group, Ostriker and Gunn ${ }^{17,18}$ have done a model considering rotating white dwarfs as the source. On that case the source would be fast and compact, requiring a more realistic equation of state, which already have been derived by Hamada and Salpeter. ${ }^{19}$ Later Usov, ${ }^{20,21}$ has shown that WD can produce pairs of electron-positron $e^{ \pm}$in its magnetosphere like a neutron star pulsar. Zhang and $\mathrm{Gil}^{22}$ interpret the transient radio source, GCRT J1745-3009, as a white dwarf pulsar with a period of 77.13 minutes. These works always have explored the possibility of white dwarf pulsar, essentially, from the theoretical point of view. The confirmation of this hypothesis happened in a very recent discovery with the source AR Scorpii (AR Sco's), which is a binary system with a pulsating white dwarf. ${ }^{3,4}$ The WD in AR Sco's has range of mass $0.81 M_{\odot}<M 1<1.29 M_{\odot}$ and pulses with quite short period of 1.97 minutes. This pulse reflects the spin of the WD, slowing down on a $10^{7}$-year timescale. The white dwarf pulsar broadband spectrum is characteristic of synchrotron radiation ${ }^{23}$ and produces radiation from X-ray to radio wavelengths, typically of neutron star pulsars. This rapidly rotating magnetized WD would mimic the neutron star pulsars as pointed out by Geng et al. ${ }^{24}$ Another specific example is 


\section{E. Otoniel et al.}

AE Aquarii, the first white dwarf pulsar (an intermediate polar WD), very fast with a short period $P=33 \mathrm{~s}$, and spinning down at a rate $\dot{P}=5.64 \times 10^{-14} \mathrm{~s} / \mathrm{s}$. The luminosity of this source and the nature of pulse hard X-ray emission detected with SUZAKU space telescope ${ }^{25,26}$ can be explained in terms of spin-powered pulsar mechanism $^{27,28}$ and emit detectable high-energy radiation. ${ }^{29-31}$

The spin-powered pulsar model considers conversion of rotational energy into electromagnetic energy, i.e., the loss of star rotational energy provides the electromagnetic power. A dipole moment $\mu$ with an orientation $\alpha$ in the rotation axis that rotates with an angular frequency $\Omega$. Such configuration in an infinity referential frame radiates with a rate

$$
\dot{E}_{\text {dip }}=\frac{2 \mu^{2} \Omega^{4} \sin ^{2} \alpha}{3 c^{3}}
$$

where $c$ is the speed of light. The loss of pulsar rotational energy is: ${ }^{32}$

$$
\dot{E}_{\mathrm{rot}}=I \Omega \dot{\Omega}+\frac{1}{2} \dot{I} \Omega^{2} \approx I \Omega \dot{\Omega} .
$$

Combining (7) and (8) we obtain the surface magnetic field at the equator as: ${ }^{33}$

$$
B_{\mathrm{s}}=B_{\mathrm{p}} / 2=\left(\frac{3 c^{3} I}{8 \pi^{2} R^{6}} P \dot{P}\right)^{1 / 2}
$$

with period $P=2 \pi / \Omega$, and $\dot{P}=d P / d t$. We see that the value of the magnetic field is inferred from $\sqrt{P \dot{P}}$ that are measures obtained by astronomical observations, and two physical quantities, the moment of inertia $I$ and the star radius $R$ that depend how the mass-energy density is distributed inside the star. Considering that star has spherical symmetry, we can solve the Tolman-Oppenheimer-Volkoff equation for specific equations of states that are quite different for neutron stars or white dwarfs. However, for high magnetic fields, as was shown by Coelho et al., ${ }^{34}$ they induce a breaking of spherical symmetry. The intrinsic geometry of this system is axisymmetric rather than spherical. Some recent works assumed a cylindrical metric and an anisotropic energy-momentum tensor for the source in a first approximation. ${ }^{35,36}$

In our case we solve fully general relativity coupled to electromagnetic source. This method resides within the named $3+1$ formalism $^{37}$ that is an approach to general relativity, where we write a system of second order differential equations into a first order metric tensor.

\section{Stellar Structure Equations}

The numerical technique used here to study axisymmetric magnetic fields was first applied to neutron stars, and more recently in Ref. ${ }^{38}$ The same formalism was used to study rotating and magnetized white dwarfs in Ref. ${ }^{39}$ Following this work, we build stellar equilibrium configurations by solving the Einstein-Maxwell field equations in a fully general relativistic approach. Below we show the basic electromagnetic equations which, combined with the gravitational equations, are solved 
numerically by using a spectral method. In this context, the stress-energy tensor $T_{\alpha \beta}$ is composed by the matter and the electromagnetic source terms:

$$
T_{\alpha \beta}=(e+p) u_{\alpha} u_{\beta}+p g_{\alpha \beta}+\frac{1}{\mu_{0}}\left(F_{\alpha \mu} F_{\beta}^{\mu}-\frac{1}{4} F_{\mu \nu} F^{\mu \nu} \mathrm{g}_{\alpha \beta}\right),
$$

with $F_{\alpha \mu}$ being the antisymmetric Faraday tensor defined as $F_{\alpha \mu}=\partial_{\alpha} A_{\mu}-\partial_{\mu} A_{\alpha}$, where $A_{\mu}$ is the non-zero components of electromagnetic four-potential, $A_{\mu}=$ $\left(A_{t}, 0,0, A_{\phi}\right)$, a time dependent poloidal field is considered. Total energy density of the system is $e$, the pressure is denoted by $p, u_{\alpha}$ is the fluid 4-velocity, and the metric tensor is $g_{\alpha \beta}$. The first term in Eq. (10) represents the isotropic matter contribution to the energy momentum-tensor, while the second term is the anisotropic electromagnetic field contribution.

The metric tensor in this axisymmetric spherical-like coordinates $(r, \theta, \phi)$ can be expressed as:

$$
d s^{2}=-N^{2} d t^{2}+\Psi^{2} r^{2} \sin ^{2} \theta\left(d \phi-N^{\phi} d t\right)^{2}+\lambda^{2}\left(d r^{2}+r^{2} d \theta^{2}\right),
$$

with $\mathrm{N}, N^{\phi}, \Psi$ and $\lambda$ being functions of the coordinates $(r, \theta) \cdot{ }^{40,41}$

The equation of motion for a star endowed with magnetic fields reads:

$$
H(r, \theta)+\nu(r, \theta)+M(r, \theta)=\text { const, }
$$

where $H(r, \theta)$ is the enthalpy heat function defined in terms of the baryon number density $n$,

$$
H=\int_{0}^{n} \frac{1}{e\left(n_{1}\right)+p\left(n_{1}\right)} \frac{d P}{d n}\left(n_{1}\right) d n_{1} .
$$

The quantity $\nu(r, \theta)$ in Eq. (11) is defined as $\nu=\ln N$, and the magnetic potential $M(r, \theta)$ is given by:

$$
M(r, \theta)=M\left(A_{\phi}(r, \theta)\right) \equiv-\int_{A_{\phi}(r, \theta)}^{0} f(x) \mathrm{d} x,
$$

with $f(x)$ being the current function, and $A_{\phi}$ is the poloidal component of the vector potential. The magnetic stellar models are obtained by assuming a constant value $f_{0}$ for the dimensionless current functions. ${ }^{39}$ Other choices for $f(x)$ are possible, but the general conclusions remain the same.

\section{Results}

In this section, we discuss the effects of strong magnetic fields on the global properties of stationary white dwarfs, such as the mass-radius diagram. In addition, we make use of an equation of state for the matter that includes both the electron-ion interactions and the latest experimental atomic mass data. The instabilities related to the microphysics (pycnonuclear fusion and $\beta$ inverse decay reactions) showed in 


\section{E. Otoniel et al.}

the figure are fundamental since they restrict equilibrium configurations and constrain the maximum magnetic fields that these stars can have in their interior. ${ }^{16,42}$ In addition, magnetic profiles were obtained by assuming the constant magnetic dipole moments $\mu$, defined as ${ }^{40,41}$

$$
\frac{2 \mu \cos \theta}{r^{3}}=\left.B(r)\right|_{r \rightarrow \infty}
$$

which is simply the radial magnetic field component (the orthonormal one) of a magnetic dipole seen by an observer at infinity.

In Fig. 1 we present carbon white dwarf equations of state with and without lattice contributions. The black lines (no lattice contribution) and red dashed line (with lattice contribution) are for white dwarf matter with zero magnetic field $(B=$ $0)$. In the right panel we show a zoom of the high energy behaviour of the EoS. We can see that the presence of the lattice contribution become the EoS softer, once that for the same value of the pressure the mass density is large when electron-ion interaction is taken into account.

According to Eq. (14), the magnetic field at the star surface is determined by the magnetic dipole moment $\mu$ and the stellar radius. In Fig. 2, we show the mass-radius relationship for magnetized white dwarfs for different magnetic dipole moments $\mu$. From Fig. 2, we see that stars become bigger for larger values of $\mu$. Thus, for smaller $\mu$ stars are more compact, and because of that the maximum magnetic field strength at the center of white dwarf is larger for smaller $\mu$, as we show in Fig. 2.
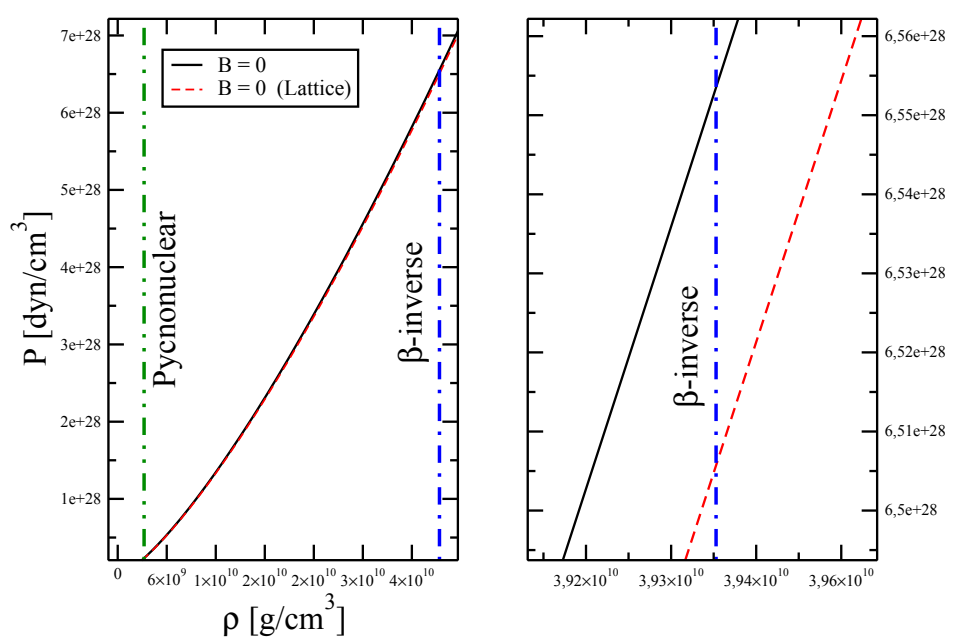

Fig. 1. Models for the equation of state of carbon white dwarfs with and without lattice contributions, at $B=0$. In the right panel we show a zoom of the high energy behaviour of the EoS. 


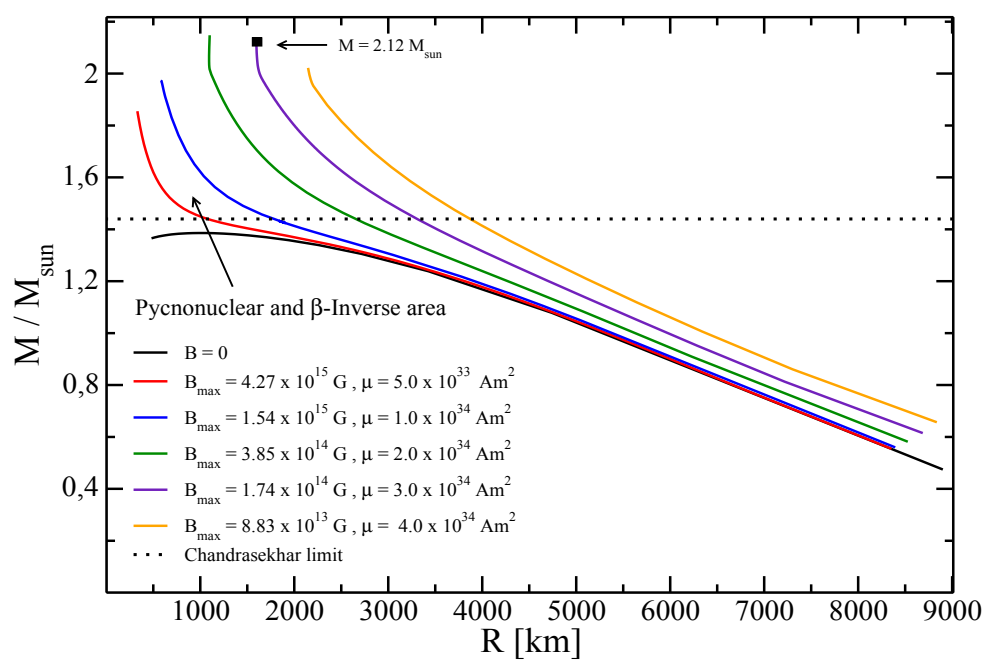

Fig. 2. (Color online) Mass-radius relationship for magnetized white dwarfs assuming different magnetic dipole moments, $\mu$. We also show the maximum Magnetic field strenght at the star center.

\section{Conclusions}

We have shown that the masses of white dwarfs increase up to $M=2.12 M_{\odot}$ with a corresponding magnetic dipole moment of $\mu=3.0 \times 10^{34} \mathrm{Am}^{2}$ if microphysical instabilities are considered. This star has an equatorial radius of $\sim 1596 \mathrm{~km}$ with magnetic fields of $B_{c}=1.74 \times 10^{14} \mathrm{G}$ and $B_{s}=3.6 \times 10^{13} \mathrm{G}$ at the center and at the stellar surface, respectively. For this white dwarf, the ratio between the magnetic pressures and the matter pressure is 0.867 . In this work, the maximum magnetic field found is an order of magnitude smaller than in Ref. ${ }^{39}$ Our results show that the surface magnetic field, $B_{s}$, is about one order of magnitude smaller than the magnetic field reached at the stellar center, $B_{c}$.

\section{Acknowledgments}

We would like to thank the organizing committee of IWARA2016 for the nice scientific atmosphere created in Gramado, Brazil. We also thank the Brazilian agencies CAPES, CNPq and FAPESP under thematic project 13/26258-4 for financial support. B. Franzon acknowledges support from CNPq/Brazil, DAAD and HGS-HIRe for FAIR. S. Schramm acknowledges support from the HIC for FAIR LOEWE program. F. Weber is supported by the National Science Foundation (USA) under Grant PHY-1411708. 
E. Otoniel et al.

\section{References}

1. S. O. Kepler, I. Pelisoli, S. Jordan, S. J. Kleinman, D. Koester, B. K. V. P. B. G. Castanheira, A. Nitta, J. E. S. Costa, D. E. Winget, A. Kanaan, and L. Fraga, Monthly Notices of the Royal Astronomical Society 429(3), 2934 (2013).

2. S. O. Kepler, A. D. Romero, I. Pelisoli, and G. Ourique, arXiv:1702.01134 [astroph.SR], No. 2 (2017)

3. T. R. Marsh, B. T. Gänsicke, S. Hümmerich, F.-J. Hambsch, K. Bernhard, C. Lloyd, E. Breedt, E. R. Stanway, D. T. Steeghs, S. G. Parsons, O. Toloza, M. R. Schreiber, P. G. Jonker, J. van Roestel, T. Kupfer, A. F. Pala, V. S. Dhillon, L. K. Hardy, S. P. Littlefair, A. Aungwerojwit, S. Arjyotha, D. Koester, J. J. Bochinski, C. A. Haswell, P. Frank and P. J. Wheatley, Nature, 537, 374 (2016).

4. D. A. H. Buckley, P. J. Meintjes, S. B. Potter, T. R. Marsh, and B. T. Gänsicke, Nature Astronomy 1(1), 0029 (2017).

5. F. Weber, Progress in Particle and Nuclear Physics 54(3), 193 (2005).

6. M. Malheiro, J. A. Rueda, and R. Ruffini, Publications of the Astronomical Society of Japan 64(6), 56 (2012).

7. R. C. Duncan and C. Thompson, The Astrophysical Journal 392(6), p. L9 (1992).

8. R. Turolla, S. Zane, and A. Watts, Reports on Progress in Physics 78(7), 116901 (2015).

9. S. Mereghetti, J. A. Pons, and A. Melatos, Space Science Reviews 191(10), 315 (2015)

10. S. A. Olausen and V. M. Kaspi, The Astrophysical Journal Supplement Series 212(5), $6(2014)$

11. J. G. Coelho and M. Malheiro, SGRs and AXPs as White Dwarf Pulsars, No. 3 (AIP Publishing, College Park, 2013).

12. M. Malheiro and J. G. Coelho, arXiv:130\%.5074 [astro-ph], No. 7 (2013).

13. B. Mukhopadhyay and A. Rao, Journal of Cosmology and Astroparticle Physics 2016, 007 (2016).

14. J. G. Coelho and M. Malheiro, Publications of the Astronomical Society of Japan 66(2), 14 (2014)

15. R. V. Lobato, M. Malheiro, and J. G. Coelho, International Journal of Modern Physics D 25(3), 1641025 (2016)

16. N. Chamel, A. F. Fantina, and P. J. Davis, Physical Review D 88(10), 081301 (2013)

17. J. Ostriker, Nature 217(3), 1227 (1968)

18. J. E. Gunn and J. P. Ostriker, Nature 221(2), 454 (1969)

19. T. Hamada and E. E. Salpeter, The Astrophysical Journal 134(11), 683 (1961)

20. V. V. Usov, Soviet Astronomy Letters, 14(4), 258 (1988).

21. V. V. Usov, The Astrophysical Journal 410(6), 761 (1993).

22. B. Zhang and J. Gil, The Astrophysical Journal 631(10), L143 (2005).

23. J. I. Katz, The Astrophysical Journal 835(2), 150 (2017).

24. J.-J. Geng, B. Zhang, and Y.-F. Huang, The Astrophysical Journal 831(10), L10 (2016).

25. Y. Terada, M. Ishida, K. Mukai, T. Dotani, K. Makishima, S. Naik, T. Hayashi, S. Okada, R. Nakamura, and T. Enoto, Advances in Space Research 41(1), 512 (2008).

26. B. Oruru and P. J. Meintjes, Monthly Notices of the Royal Astronomical Society 421(4), 1557 (2012).

27. N. R. Ikhsanov, Astronomy and Astrophysics 338(10), 521 (1998).

28. B. Oruru and P. J. Meintjes, EPJ Web of Conferences 64(1), 07003 (2014).

29. D. L. Caceres, S. M. de Carvalho, J. G. Coelho, R. C. R. de Lima, and J. A. Rueda, Monthly Notices of the Royal Astronomical Society 465(11), 4434 (2017). 
30. R. V. Lobato, J. Coelho, and M. Malheiro, Journal of Physics: Conference Series 630(7), 012015 (2015).

31. R. V. Lobato, J. G. Coelho, and M. Malheiro, Radio pulsar death lines to SGRs/AXPs and white dwarfs pulsars, in The Second Icranet César Lattes Meeting: Supernovae, Neutron Stars and Black Holes, (AIP Publishing, Rio de Janeiro, Niterói, João Pessoa, Recife, Fortaleza, 2015).

32. S. L. Shapiro and S. A. Teukolsky, Black Holes, White Dwarfs and Neutron Stars: The Physics of Compact Objects, No. 11 (John Wiley \& Sons, 2008).

33. A. Ferrari and R. Ruffini, The Astrophysical Journal Letters 158(11), L71 (1969).

34. J. G. Coelho, R. M. Marinho, M. Malheiro, R. Negreiros, D. L. Cáceres, J. A. Rueda, and R. Ruffini, The Astrophysical Journal 794(10), 86 (2014).

35. D. M. Paret, J. E. Horvath, and A. P. Martínez, arXiv:1407.2280 [astro-ph], No. 7 (2014).

36. D. M. Paret, J. E. Horvath, and A. P. Martínez, Research in Astronomy and Astrophysics 15(10), 1735 (2015).

37. E. Gourgoulhon, $3+1$ Formalism in General Relativity, Lecture Notes in Physics, Vol. 846, No. 3 (Springer Berlin Heidelberg, Berlin, Heidelberg, 2012).

38. B. Franzon, V. Dexheimer, and S. Schramm, Monthly Notices of the Royal Astronomical Society 456(3), 2937 (2016).

39. B. Franzon and S. Schramm, Physical Review D 92(10), 083006 (2015).

40. S. Bonazzola, E. Gourgoulhon, M. Salgado, and J. A. Marck, Astronomy and Astrophysics 278(11), 421 (1993).

41. M. Bocquet, S. Bonazzola, E. Gourgoulhon, and J. Novak, Astronomy and Astrophysics 301(3), 757 (1995).

42. E. Otoniel, B. Franzon, M. Malheiro, S. Schramm, and F. Weber, arXiv:1609.05994 [astro-ph.SR], No. 9 (2016). 